\title{
A One-Atom Laser in a Regime of Strong Coupling
}

\author{
J. McKeever, A. Boca, A. D. Boozer, J. R. Buck, and H. J. Kimble \\ Norman Bridge Laboratory of Physics 12-33, California Institute of Technology, Pasadena, CA 91125
}

(Dated: October 25, 2018)

\begin{abstract}
Although conventional lasers operate with a large number of intracavity atoms, the lasing properties of a single atom in a resonant cavity have been theoretically investigated for more than a decade $[1,2,3,4,[5,6,7,8,4,10,11]$. In this Letter we report the experimental realization of such a one-atom laser operated in a regime of strong coupling. Our experiment exploits recent advances in cavity quantum electrodynamics that allow one atom to be isolated in an optical cavity in a regime for which one photon is sufficient to saturate the atomic transition [12]. In this regime the observed characteristics of the atom-cavity system are qualitatively different from those of the familiar many atom case. Specifically, we present measurements of intracavity photon number versus pump intensity that exhibit "thresholdless" behavior, and infer that the output flux from the cavity mode exceeds that from atomic fluorescence by more than tenfold. Observations of the second-order intensity correlation function $g^{(2)}(\tau)$ demonstrate that our one-atom laser generates manifestly quantum (i.e., nonclassical) light that exhibits both photon antibunching $g^{(2)}(0)<g^{(2)}(\tau)$ and sub-Poissonian photon statistics $g^{(2)}(0)<1$.
\end{abstract}

An important trend in modern science is to push macroscopic physical systems to ever smaller sizes, eventually into the microscopic realm. Lasers are one important example of this progression, having moved from table-top systems to microscopic devices that are ubiquitous in science and technology. However, even over this remarkable span of implementations, lasers are typically realized with large atom and photon numbers in a domain of weak coupling for which individual quanta have negligible impact on the system dynamics. Usual laser theories therefore rely on system-size expansions in inverse powers of critical atom and photon numbers $\left(N_{0}, n_{0}\right) \gg 1$, and arrive at a consistent form for the laser characteristics [13, 14, 15, 16, 17]. By contrast, over the past twenty years, technical advances on various fronts have pushed laser operation to regimes of ever smaller atom and photon number, pressing toward the limit of strong coupling for which $\left(N_{0}, n_{0}\right) \ll 1[18]$. Significant milestones include the realization of one and two-photon micromasers [19, 20, 21], as well as novel microlasers in atomic and condensed matter systems [22, 23, 24].

In this march toward ever smaller systems, an intriguing possibility is that a laser might be obtained with a single atom in an optical cavity, as was considered in the

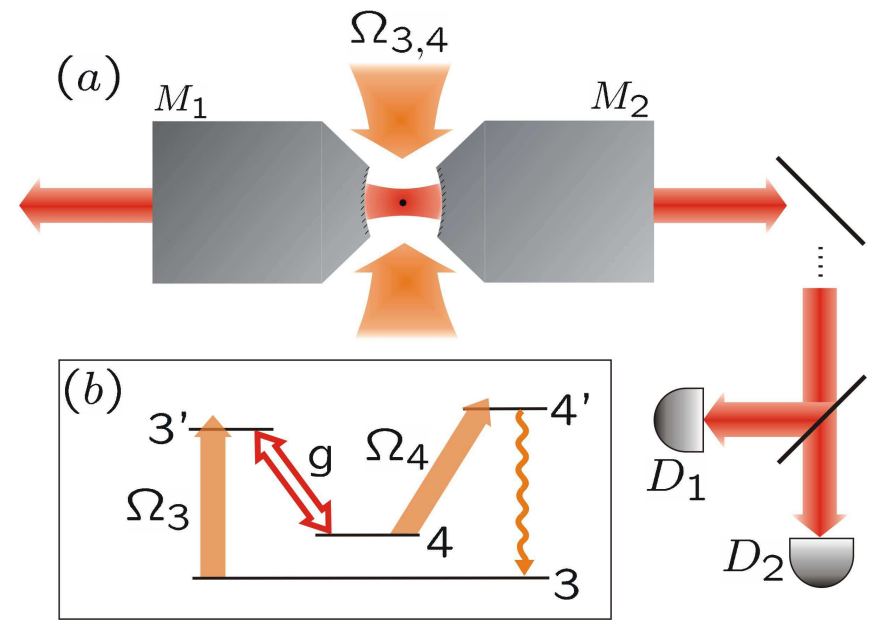

FIG. 1: A simplified schematic of the experiment. (a) A Cesium atom (black dot) is trapped inside a high-finesse optical cavity formed by the curved, reflective surfaces of mirrors $M_{1,2}$. Light generated by the atom's interaction with the resonant cavity mode propagates as a Gaussian beam to single-photon detectors $D_{1,2}$. (b) The relevant transitions involve the $6 S_{1 / 2}, F=3,4 \leftrightarrow 6 P_{3 / 2}, F^{\prime}=3^{\prime}, 4^{\prime}$ levels of the $D_{2}$ line at $852.4 \mathrm{~nm}$ in atomic Cesium. Strong coupling at rate $g$ is achieved for the lasing transition $F^{\prime}=3^{\prime} \rightarrow F=4$ near a cavity resonance. Pumping of the upper level $F^{\prime}=3^{\prime}$ is provided by the field $\Omega_{3}$, while recycling of the lower level $F=4$ is achieved by way of the field $\Omega_{4}\left(4 \rightarrow 4^{\prime}\right)$ and spontaneous decay back to $F=3$. Decay $\left(3^{\prime}, 4^{\prime}\right) \rightarrow(3,4)$ is also included in our model. Relevant cavity parameters are length $l_{0}=42.2 \mu \mathrm{m}$, waist $w_{0}=23.6 \mu \mathrm{m}$, and finesse $\mathcal{F}=4.2 \times 10^{5}$ at $\lambda_{D_{2}}=852 \mathrm{~nm}$.

seminal work of $\mathrm{Mu}$ and Savage 1] and has since been extensively analyzed [2, 3, 4, [5, 6, 7, 8, 9, 10, 11]. In this Letter we report an experiment that advances this quest to its conceptual limit, namely the operation of a one-atom laser in a regime for which $\left(N_{0}, n_{0}\right) \ll 1$. We describe measurements of the operating characteristics of the atom-cavity system, including the light output as a function of pumping strength. Significantly, the light emission is observed to exhibit photon antibunching and is manifestly quantum in character, in contrast to the light from conventional lasers, thereby enabling diverse applications in quantum optics and quantum information science.

As illustrated in Fig. 10 our experiment consists of a single Cesium atom trapped in a far-off-resonance trap (FORT) within a high-finesse optical cavity [12, 25]. The lasing transition $6 P_{3 / 2}, F^{\prime}=3^{\prime} \rightarrow 6 S_{1 / 2}, F=4$ is nearly 
resonant with and strongly coupled to a single mode of this cavity. The coupling is parameterized by the Rabi frequency $2 g_{0}$ for a single quantum of excitation, and the atom and field have amplitude decay rates $\gamma$ and $\kappa$, respectively. The upper level $F^{\prime}=3^{\prime}$ is pumped by the external drive $\Omega_{3}$, while effective decay of the lower level $F=4$ takes place via the combination of the drive $\Omega_{4}$ and decay $\gamma_{34}, 4 \rightarrow 4^{\prime} \rightarrow 3$. In essential character this system is analogous to a Raman scheme with pumping $3 \rightarrow 3^{\prime}$, lasing $3^{\prime} \rightarrow 4$, and decay $4 \rightarrow 3$. Of particular relevance to our work are the detailed treatments of the ion-trap laser by Walther and colleagues [6, 7, 8, 9].

We emphasize that a "one-and-the-same" atom laser as illustrated in Fig. 1 is quite distinct from "single-atom" micro-masers 19, 20, 21] and lasers 22] for which steady state is reached through the incremental contributions of many atoms that transit the cavity, even if one by one [19, 20] or few by few 22]. By contrast, in our experiment steady state is reached with one-and-the-same atom over a time interval $\delta t \sim 10^{-7} \mathrm{~s}$ that is much shorter than the trap lifetime $\Delta t \sim 0.05 \mathrm{~s}$. Our pumped atom-cavity system provides a continuous source of nonclassical light as a Gaussian beam for the entire duration that an atom is trapped.

Because conventional lasers operate in the limit $\left(N_{0}, n_{0}\right) \gg 1$, there is a generic form associated with the laser threshold in the transition from nonlasing to lasing action that is independent of the model system [13, 26]. However, as the system size is reduced, the sharpness of the laser "turn on" is lost, with then no clear consensus about how to define the lasing threshold [26]. Well into the regime of strong coupling $\left(N_{0}, n_{0}\right) \ll 1$, even the familiar qualitative characteristics of a laser (e.g., the statistical properties of the output light) are profoundly altered, leaving open the question of how to recognize a laser in this new regime.

To address this question, we have carried out extensive theoretical analyses for a four-state model based upon Fig. 1(b) for parameters relevant to our experiment. A synopsis of relevant results from this work is given in the Supplementary Information, with the full treatment presented in Ref. [27]. In brief, the steady state solutions obtained from a semiclassical theory exhibit familiar characteristics of conventional lasers, including a clearly defined laser threshold and population inversion. The condition $C_{1} \gg 1$ is required to observe threshold behavior for one atom pumped inside the resonator, where for our experiment the cooperativity parameter $C_{1}=1 / N_{0} \simeq 12$. By contrast, the fully quantum analysis for the four-state model results in qualitatively different characteristics. In particular, the input-output relationship for the mean intracavity photon number $\bar{n}$ versus the pump intensity $I_{3}=\left(\Omega_{3} / 2 \gamma\right)^{2}$ has several key features to be compared with experimental results presented below, namely the immediate onset of emission ("thresholdless" behavior), and the saturation and eventual quenching of the output.

Our actual experiment is somewhat more complex than indicated by the simple drawing in Fig. 1 with many of the technical aspects described in more detail in Refs. 12, 25. In brief, the principal cavity QED (cQED) parameters of our system are $g_{0} / 2 \pi=16 \mathrm{MHz}$, $\kappa / 2 \pi=4.2 \mathrm{MHz}$, and $\gamma / 2 \pi=2.6 \mathrm{MHz}$, where $g_{0}$ is based upon the reduced dipole moment for the $6 S_{1 / 2}, F=4 \leftrightarrow$ $6 P_{3 / 2}, F^{\prime}=3^{\prime}$ transition in atomic Cs. Strong coupling is thereby achieved $\left(g_{0} \gg(\kappa, \gamma)\right)$, resulting in critical photon and atom numbers $n_{0} \equiv \gamma^{2} /\left(2 g_{0}^{2}\right) \simeq 0.013$, $N_{0} \equiv 2 \kappa \gamma / g_{0}^{2} \simeq 0.084$.

Atoms are trapped in the cavity by means of a faroff-resonance trap (FORT) [28] with wavelength $\lambda_{F}=$ $935.6 \mathrm{~nm}$, which is matched to a $\mathrm{TEM}_{00}$ mode along the cavity axis. For all experiments herein, the trap depth is $U_{0} / k_{B}=2.3 \mathrm{mK}(47 \mathrm{MHz})$. The FORT has the important feature that the potential for the atomic centerof-mass motion is only weakly dependent on the atom's internal state [12].

After the trap-loading stage (as described in the section on Methods), the transverse $\Omega_{3,4}$ fields are switched to pump and recycle the atomic population in the fashion depicted in Fig. 1(b). Two examples of the resulting output counts versus time are shown in Fig. 2 By averaging traces such as these, we arrive at an average signal level versus time, as shown in the inset to Fig. 22(a). Typical lifetimes for a trapped atom in the presence of the driving $\Omega_{3,4}$ fields are $50-100 \mathrm{~ms}$, which should be compared to the lifetimes of $2-3 \mathrm{~s}$ recorded in the absence of these fields 12]. Significantly, the approximately exponential decay of the signal with time does not result from a time-dependent diminution of the flux from single trapped atoms, but rather from the average of many events each of a variable duration. That is, for a given set of external control parameters, each atom gives a reasonably well-defined output flux over the time that it is trapped.

For a fixed set of operating conditions, we collect a set of 60-300 traces as in Fig. 2] determine the average output flux for each trace, and find the mean and variance, as well as the trap lifetime for the set. Figure 3 displays a collection of such measurements for the mean intracavity photon number $\bar{n}$ as a function of the dimensionless pump intensity $x$, scaled in units of the fixed recycling intensity (see section on Methods). More precisely, the parameter $x$ is the ratio of measured intensities, and can be written as $x \equiv(7 / 9)\left(I_{3} / I_{4}\right)$, where $I_{3,4} \equiv\left(\Omega_{3,4} / 2 \gamma\right)^{2}$. The factor of $(7 / 9)$ is needed because the the two transitions have different dipole moments. For these measurements, we estimate that the incoherent sum of intensities of the four $\Omega_{4}$ beams is about $50 \mathrm{~mW} / \mathrm{cm}^{2}$, which corresponds to $I_{4} \sim 13$. The output count rate at detectors $D_{1,2}$ is converted to intracavity photon number using the known propagation and detection efficiency $\xi=0.05$.

Important features of the data shown in Fig. Binclude the prompt onset of output flux $\kappa \bar{n}$ emerging through the cavity mirrors $M_{1,2}$ as the pump intensity $I_{3}$ is increased from zero. In a regime of strong coupling, the atom-cavity system behaves as a "thresholdless" device. 


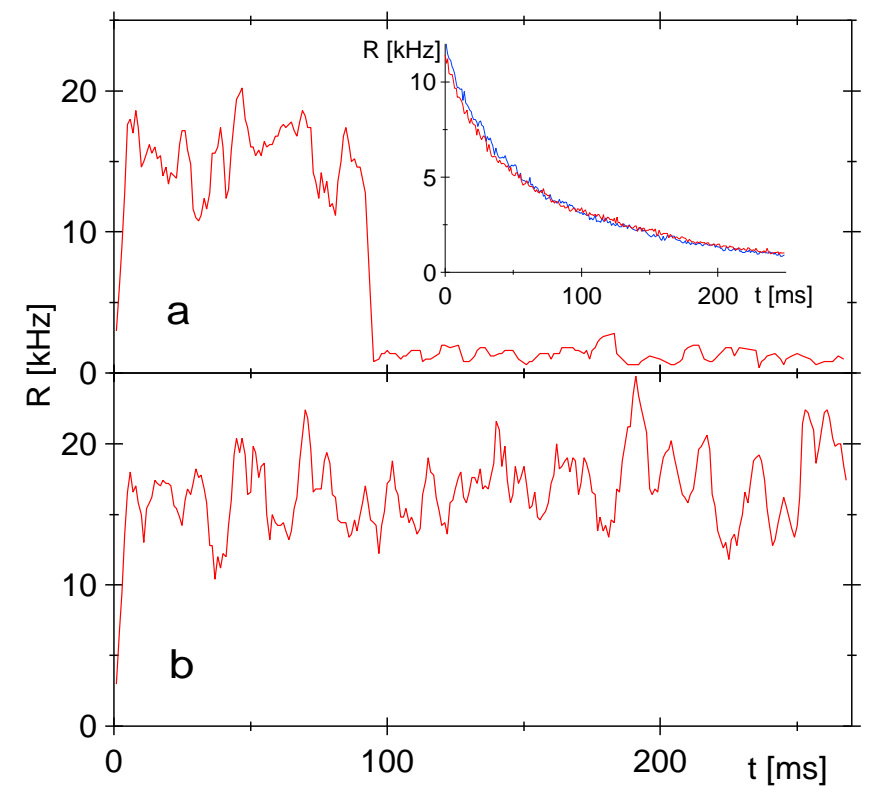

FIG. 2: Total counting rate $R$ recorded by detectors $D_{1,2}$ is displayed as a function of time for two separate trapped atoms, with the counts summed over $5 \mathrm{~ms}$ bins. At $t=0$, the $\Omega_{3,4}$ fields are switched to predetermined values of intensity and detuning. In (a), the atom is trapped for $t \simeq 90 \mathrm{~ms}$ before escaping, with the background level due to scattered light from the $\Omega_{3,4}$ fields and detector dark counts evident as the residual output at later times. In (b), the atom (atypically) remains trapped for the entire observation cycle $\simeq 270 \mathrm{~ms}$ and then is dumped. The inset in (a) displays $R$ versus time obtained by averaging about 400 such traces. Two cases are shown; in one, the number of atoms delivered to the cavity mode has been diminished by about 2 -fold. Since the curves are nearly identical, we conclude that cases with $N>1$ atom play a negligible role. The overall detection efficiency $\xi=$ 0.05 from intracavity photon to a detection event at $D_{1,2}$ is made up of the following factors: $\eta=0.60$ cavity escape efficiency, $T=0.50$ for only mirror $M_{2}$ output, $\zeta=0.33$ propagation efficiency from $M_{2}$ to $D_{1,2}, \alpha=0.5$ detection quantum efficiency at $D_{1,2}$.

With further increases in pump intensity $I_{3}$, the output flux saturates at a maximum value $\kappa \bar{n}_{\max }$ around $x \simeq 0.1$. We attribute this behavior to a bottleneck associated with the recycling of population $4 \rightarrow 4^{\prime} \rightarrow 3$, with the rate limiting step in the recycling process being spontaneous decay $4^{\prime} \rightarrow 3$ at rate $\gamma_{34}$ in the limit of large Rabi frequency $\Omega_{4} \gg \gamma$. For a single intracavity atom, quanta can be deposited into the cavity mode no faster than the maximum recycling rate. As the pump level $I_{3}$ is increased beyond $x \sim 1$, the output flux $\kappa \bar{n}$ gradually drops, presumably due to splitting of the pumped excited state $F^{\prime}=3^{\prime}$ by the Autler-Townes effect, although this is still under investigation. Heating of the atomic motion at higher pump levels is certainly a concern as well; however our simulations, which do not incorporate atomic motion, show the same trend as in Fig. 3 [27].

Beyond these considerations, we have also undertaken
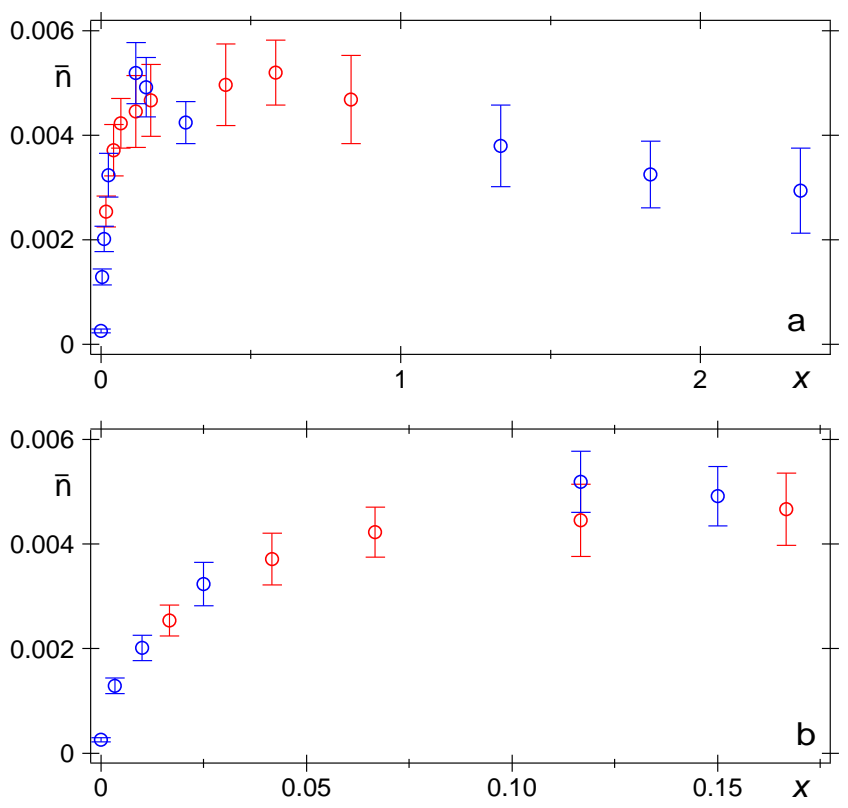

FIG. 3: The intracavity photon number $\bar{n} \pm \sigma_{n}$ inferred from measurements as in Fig. 2 is plotted as a function of dimensionless pump intensity $x \equiv(7 / 9)\left(I_{3} / I_{4}\right)$ for fixed $I_{4}=13$ over two ranges of pump level $x$. (a) $\bar{n}$ versus $x$ is shown over the entire range $x=0$ to 2.33 recorded in our measurements (b) An expanded scale displays $\bar{n}$ for small $x$. The immediate onset of emission supports the conclusion of "thresholdless" lasing. The two independent sets of measurements (red and blue points) agree reasonably well.

extensive theoretical analyses based both upon the fourstate model shown in Fig. 1 as well as on the full set of Zeeman states for each of the levels $F=3,4$ and $F^{\prime}=3^{\prime}, 4^{\prime}$ and two cavity modes, one for each of two orthogonal polarizations [27. These analyses are in reasonable accord with the principal features of the data in Fig. 3 Moreover, our quantum simulations support the conclusion that the range of coupling values $g$ that contribute to our results is restricted roughly to $0.5 g_{0} \lesssim g \lesssim g_{0}$. Furthermore, the simulations yield information about the atomic populations, from which we deduce that the rate of emission from the cavity $\kappa \bar{n}$ exceeds that by way of fluorescent decay $3^{\prime} \rightarrow 4, \gamma_{43^{\prime}}\left\langle\sigma_{3^{\prime} 3^{\prime}}\right\rangle$, by roughly tenfold over the range of pump intensity $I_{3}$ shown in Fig. 3 where $\left\langle\sigma_{3^{\prime} 3^{\prime}}\right\rangle$ is the steady state population in level $3^{\prime}$.

To investigate the quantum statistical characteristics of the light emerging in the $\mathrm{TEM}_{00}$ mode of the cavity output, we probe the photon statistics of the light by way of the two single-photon detectors $D_{1,2}$ illustrated in Fig. 1] From the cross-correlation of the resulting binned photon arrival times and the mean counting rates of the signals and the background, we construct the normalized intensity correlation function (see the Supplementary Information)

$$
g^{(2)}(\tau)=\frac{\langle: \hat{I}(t) \hat{I}(t+\tau):\rangle}{\langle: \hat{I}(t):\rangle^{2}}
$$



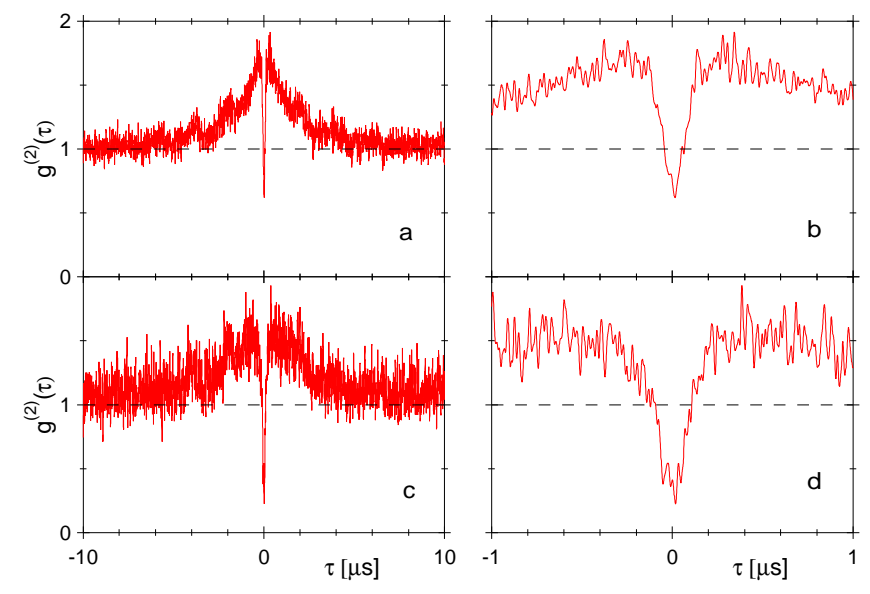

FIG. 4: The intensity correlation function $g^{(2)}(\tau)$ is given for two values of the pump intensity in (a-d), where (b,d) show the central features of $(\mathrm{a}, \mathrm{c})$ over a smaller range of $\tau$. Panels $(\mathrm{a}, \mathrm{b})$ are for pump intensity parameter $x=0.83$, whereas for (c,d) $x=0.17$. Note that the light exhibits sub-Poissonian photon statistics and antibunching. All traces have been "smoothed" by convolution with a Gaussian function of width $\sigma=5 \mathrm{~ns}$.

where the colons denote normal and time ordering for the intensity operators $\hat{I}$ [15]. Over the duration of the trapping events, we find no evidence that $\langle: \hat{I}(t):\rangle$ is a function of $t$, although we do not have sufficient data to confirm quantitatively stationarity of the underlying processes.

Examples of two measurements for $g^{(2)}(\tau)$ are given in Fig. 4(a-d). In Fig. 4(a,b), we again have $I_{4} \simeq 13$ and the pump intensity $I_{3}$ is set for operation with $x \simeq 0.83$ well beyond the "knee" in $\bar{n}$ versus $x$, while in $(\mathrm{c}, \mathrm{d})$, the pump level is decreased to $x \simeq 0.17$ near the peak in $\bar{n}$. Significantly, in each case these measurements demonstrate that the light from the atom-cavity system is manifestly quantum (i.e., nonclassical) and exhibits photon antibunching $g^{(2)}(0)<g^{(2)}(\tau)$ and sub-Poissonian photon statistics $g^{(2)}(0)<1$ 15]. The actual coincidence data $n(\tau)$ used to obtain $g^{(2)}(\tau)$ are presented in the Supplementary Information. Significantly, these data directly evidence the nonclassical character of the emitted light, with relatively minor corrections for background light required for the determination of $g^{(2)}(\tau)$.

Beyond the nonclassical features around $\tau \simeq 0, g^{(2)}(\tau)$ also exhibits excess fluctuations extending over $\tau \simeq$ $\pm 1 \mu \mathrm{s}$, with $g_{\max }^{(2)}(\tau) \simeq 1.7$. Fluctuations in the intensity of the intracavity light over these time scales are presumably related to the stochastic character of the pumping $3 \rightarrow 3^{\prime}$ and recycling $4 \rightarrow 4^{\prime} \rightarrow 3$ processes for a single, multi-state atom. Also of significance is the interplay of atomic motion and optical pumping into dark states by the $\Omega_{3,4}$ fields (which is responsible for cooling [29]), as well as Larmor precession that arises from residual ellipticity in polarization of the intracavity FORT [12, 30]. Indeed, in Fig. 4( a,c) there is a hint of an oscillatory vari- ation in $g_{\max }^{(2)}(\tau)$ with period $\tau \simeq \pm 2 \mu \mathrm{s}$. Fourier transformation of the associated coincidence data leads to a small peak at about $500 \mathrm{kHz}$, which is near to the predicted frequency for axial motion of a trapped Cs atom at the bottom of the FORT potential as well as to the Larmor frequency inferred from other measurements.

In agreement with the trend predicted by the fourstate model discussed in the Supplementary Information, $g^{(2)}(0)$ increases with increasing pump intensity, with a concomitant decrease in these nonclassical effects. Moreover, our experimental observations of $g^{(2)}(\tau)$ are described reasonably well by the results obtained from more detailed quantum simulations based upon the entire manifold of Zeeman states for the Cs atom, two cavity modes with orthogonal polarizations, and a simple model to describe the polarization gradients of the $\Omega_{3,4}$ fields [27].

The realization of this strongly coupled one-atom laser is significant on several fronts. From the perspective of the dynamics of open quantum systems, our system demonstrates the radical departures from conventional laser operation wrought by strong coupling for the quantized light-matter interaction. On a more practical level, throughout the interval when an atom is trapped (which is determined in real time), our system provides an approximately stationary source of nonclassical light in a collimated, Gaussian beam, as has been anticipated in the literature on one-atom lasers 1 , 3, 4, 5, 6, 8, 9, 10, 11], and which has diverse applications. Remaining technical issues in our work are to improve the modelling and measurements related to atomic motion, both within the FORT potential and through the polarization gradients of the $\Omega_{3,4}$ fields. We have employed our quantum simulations to calculate the optical spectrum of the light output, and have devised a scheme for its measurement.

Methods - While the atom is trapped in a standing wave FORT along the cavity axis, another set of fields (designated by $\Omega_{3,4}$ in Fig. 1) propagate in the plane transverse to the cavity axis and illuminate the region between the cavity mirrors. These fields are used not only for the pumping scheme described in association with the operation of the one-atom laser with strong coupling, but also for cooling in the trap-loading phase. Each $\Omega_{3,4}$ field consists of two orthogonal pairs of counter-propagating beams in a $\sigma^{+}-\sigma^{-}$configuration.

Unfortunately, it is difficult to calibrate accurately the intensities $I_{3,4}$ for the $\Omega_{3,4}$ beams at the location of the atom in the region between the cavity mirrors. We estimate that our knowledge of either intensity is uncertain by an overall scale factor $\simeq 2$. However, we do know the ratio of intensities much more accurately than either intensity individually, and therefore plot the data in Fig. 3] as a function of this ratio.

In the pumping stage of the experiment, the fields are tuned $10 \mathrm{MHz}$ blue of $F=3 \rightarrow F^{\prime}=3^{\prime}$ in the case of the $\Omega_{3}$ beams and $17 \mathrm{MHz}$ blue of $F=4 \rightarrow F^{\prime}=4^{\prime}$ in the case of the $\Omega_{4}$ fields. The detuning between the $3^{\prime} \rightarrow 4$ transition at $\omega_{43}$ and the cavity resonance $\omega_{C}$ is $\Delta_{C A} \equiv \omega_{C}-\omega_{43}=2 \pi \cdot 9 \mathrm{MHz}$. These detunings are 
chosen operationally in a trade-off between achieving a large cavity output flux from the $3^{\prime} \rightarrow 4$ transition while maintaining a reasonable lifetime for the trapped atom despite heating from the various fields [29]. The cavity length itself is actively stabilized with an auxiliary laser at wavelength $\lambda_{C}=835.8 \mathrm{~nm}$ that does not interfere with the trapping or the cQED interactions.

Our experimental protocol begins with the formation of a magneto-optical trap (MOT) above the cavity. After a stage of sub-Doppler cooling, the cloud of atoms is released. The $\Omega_{3,4}$ beams are then used as cooling beams (with independent settings of intensity and detuning) to load an atom into the FORT [12. About 10 atoms transit the cavity mode after each MOT drop, and the loading efficiency is set such that an atom is loaded into the FORT once every 3-10 drops. We then switch the intensities and detunings of the transverse fields $\Omega_{3,4}$ to the pumping configuration and record the cavity output by way of the single-photon detectors $D_{1,2}$ shown in Fig. [1] Each photoelectric pulse from $D_{1,2}$ is stamped with its time of detection ( $1 \mathrm{~ns}$ resolution) and then stored for later analysis, with examples of the record of output counts versus time displayed in Fig. 2
[1] Mu, Y. \& Savage, C. M. One-atom lasers. Phys. Rev. A 46, 5944-5954 (1992).

[2] Ginzel, C., Briegel, H.-J., Martini, U., Englert, B.-G. \& Schenzle, A. Quantum optical master equations: The one-atom laser. Phys. Rev. A 48, 732-738 (1993).

[3] Pellizzari, T. \& Ritsch, H. Preparation of stationary Fock states in a one-atom Raman laser. Phys. Rev. Lett. 72, 3973-3976 (1994).

[4] Pellizzari, T. \& Ritsch, H. Photon statistics of the threelevel one-atom laser. J. Mod. Opt. 41, 609-623 (1994).

[5] Horak, P., Gheri, K. M. \& Ritsch, H. Quantum dynamics of a single-atom cascade laser. Phys. Rev. A 51, 32573266 (1995).

[6] Meyer, G. M., Briegel, H.-J. \& Walther, H. Ion-trap laser. Europhys. Lett. 37, 317-322 (1997).

[7] Löffler, M., Meyer, G. M., \& Walther, H. Spectral properties of the one-atom laser. Phys. Rev. A 55, 3923-3930 (1997).

[8] Meyer, G. M., Löffler, M. \& Walther, H. Spectrum of the ion-trap laser. Phys. Rev. A 56, R1099-R1102 (1997).

[9] Meyer, G. M. \& Briegel, H.-J. Pump-operator treatment of the ion-trap laser. Phys. Rev. A 58, 3210-3220 (1998).

[10] Jones, B., Ghose, S., Clemens, J. P., Rice, P. R. \& Pedrotti, L. M. Photon statistics of a single atom laser. Phys. Rev. A 60, 3267-3275 (1999).

[11] Kilin, S. Ya. \& Karlovich, T. B. Single-atom laser: Coherent and nonclassical effects in the regime of a strong atom-field correlation. JETP 95, 805-819 (2002).

[12] McKeever, J. et al. State-Insensitive Cooling and Trapping of Single Atoms in an Optical Cavity. Phys. Rev. Lett. 90, 133602 (2003).

[13] Sargent III, M., Scully, M. O., \& Lamb, Jr., W. E. Laser Physics (Addison-Wesley, Reading Mass., 1974).

[14] Haken, H. Laser Theory (Springer Verlag, Berlin, 1984).

[15] Mandel, L. \& Wolf, E. Optical Coherence and Quantum Optics (Cambridge Univ. Press, Cambridge, 1995).

[16] Carmichael, H. J. Statistical Methods in Quantum Optics 1 (Springer-Verlag, Berlin, 1999).

[17] Gardiner, C. W. \& Zoller, P. Quantum Noise (SpringerVerlag, Berlin, 2000).

[18] Kimble, H. J. Strong interactions of single atoms and photons in cavity QED Phys. Scr. T76, 127 (1998).

[19] Raithel, G., Wagner, C., Walther, H., Narducci, L. M. \& Scully, M. O. in Cavity Quantum Electrodynamics (ed. Berman, P.) 57-121 (Academic Press, San Diego, 1994).

[20] Haroche, S. \& Raimond, J. M. in Cavity Quantum Elec- trodynamics (ed. Berman, P.) 123-170 (Academic Press, San Diego, 1994).

[21] Meystre, P. in Progress in Optics, Vol. XXX (ed. Wolf, E.) 261-355 (Elsevier Science Publishers B.V., Amsterdam, 1992).

[22] An, K. \& Feld, M. S. Semiclassical four-level single-atom laser. Phys. Rev. A 56, 1662-1665 (1997).

[23] Chang, R. K. \& Campillo, A. J. (eds) Optical Processes in Microcavities (World Scientific, Singapore, 1996).

[24] K. J. Vahala, Optical microcavities, Nature (2003).

[25] Ye, J., Vernooy, D. W. \& Kimble, H. J. Trapping of single atoms in cavity QED. Phys. Rev. Lett. 83, 4987-4990 (1999).

[26] Rice, P. R. \& Carmichael, H. J. Photon statistics of a cavity-QED laser: A comment on the laser-phasetransition analogy. Phys. Rev. A 50, 4318-4329 (1994).

[27] Boozer, A. D., Boca, A., Buck, J. R., McKeever, J. and Kimble, H. J., Comparison of Theory and Experiment for a One-Atom Laser in a Regime of Strong Coupling, Phys. Rev. A (submitted, 2003); preprint available at http://lanl.arxiv.org/archive/quant-ph.

[28] Metcalf, H. J. \& van der Straten, P. Laser Cooling and Trapping (Springer-Verlag, 1999).

[29] Boiron, D. et al. Laser cooling of cesium atoms in gray optical molasses down to $1.1 \mu \mathrm{K}$. Phys. Rev. A 53, R3734R3737 (1996) and references therein.

[30] Corwin, K. L., Kuppens, S. J. M., Cho, D. \& Wieman, C. E. Spin-polarized atoms in a circularly polarized optical dipole trap. Phys. Rev. Lett. 83, 1311-1314 (1999).

Acknowledgements We gratefully acknowledge interactions with K. Birnbaum, C.-W. Chou, A. C. Doherty, L.-M. Duan, T. Lynn, T. Northup, S. Polyakov, and D. M. Stamper-Kurn. This work was supported by the National Science Foundation, by the Caltech MURI Center for Quantum Networks, and by the Office of Naval Research.

Competing interests statement The authors declare that they have no competing financial interests.

Correspondence and requests for materials should be addressed to H.J.K. (e-mail: hjkimble@caltech.edu). 